\title{
TEXTURE CLASSIFICATION WITH ANTS
}

\author{
Arshad Hussain $^{\dagger} \quad$ Nasir Rajpoot* ${ }^{*} \quad$ Kashif Rajpoot ${ }^{\dagger}$ \\ $\dagger$ GIK Institute of Engineering Sciences \& Technology, Pakistan \\ * Department of Computer Science, University of Warwick, UK
}

Correspondence email: nasiredcs.warwick.ac.uk

\begin{abstract}
In this paper, we present a novel texture classification algorithm inspired by the self-assembling behavior of real ants when building live structures with their bodies. The proposed algorithm employs dyadic Gabor filter banks for extracting discriminant features from images containing multiple textures not known to the algorithm. The feature space is clustered using the novel ant tree clustering (ATC) algorithm based on the similarity of ants carrying the feature vectors. The results thus obtained show promise of the proposed approach.
\end{abstract}

Keywords: Texture analysis, ant colony systems, Gabor wavelets.

\section{INTRODUCTION}

Texture carries the potential to provide vital clues about image contents and objects present in images. It is hard to find a universally agreeable definition of texture in the literature. Nevertheless, texture can be described by local properties such as uniformity, density, coarseness, roughness, regularity, linearity, directionality, frequency, and phase gathered from the appearance of an image region [1]. Texture analysis has become a focussed area of research because of its utilization in numerous industrial and real-world applications [2]: contentbased image retrieval, medical image analysis, industrial inspection, remote sensing, and document processing, to name only a few.

Feature-based approach to texture classification is typically composed of two main stages: feature extraction and classification. In the first stage, characteristic attributes (features) representing the texture are gathered by means of statistical, structural, geometric, model-based, and/or filtering approaches [3]. In the second stage, a supervised or unsupervised classifier is used depending upon the nature of the application. Due to the complexity of texture analysis problem, no single approach of feature extraction or classification works for all types of texture and applications. This is the driving force behind research efforts made in this area.
In this paper, dyadic Gabor features are used because of their proven potential for describing a vast variety of textures [3]. A variant of ant tree clustering (ATC) algorithm [4] is employed for classification of the features. The algorithm is inspired by self-assembling behavior observed in biological ants when building live structures with their bodies [5]. We show that the feature data can be hierarchically organized in a tree, resembling the self-assembling behavior in real ants, where each artificial ant represents a feature vector and is attached to one of the tree branches depending on its similarity with the ants on the branches. Classification results using our ant clustering algorithm are shown to be comparable to the commonly used $k$-means clustering algorithm using the same feature data. A major disadvantage of $k$-means is that it can get stuck in local minima in high-dimensional vector spaces. The proposed algorithm uses similarity scope for partitioning the feature data and uses a probabilistic approach to avoid local minima.

\section{THE PROPOSED ALGORITHM}

\subsection{Gabor Feature Extraction}

The fundamental idea behind filtering based feature extraction is to pass the image through a bank of filters, observe their response, and compute some energy measure from subbands. The hypothesis behind this idea is that two different texture patterns have different responses to subband filtering. In this work, we employ dyadic Gabor filter banks which are essentially Gaussian bandpass filters with dyadic coverage of the radial spatial frequency range for multiple orientations. Dyadic Gabor filter banks have been shown to be related to the early vision models for mammals [3]. A number of subbands, say $n$ of them excluding the DC subband, are reverse filtered (by taking the inverse transform) to generate feature images. A local energy function is then computed corresponding to every pixel in each of the feature images yielding an $n$-dimensional feature vector (upto 32-dimensional) which is assumed to have discriminatory power for texture classification. 


\subsection{Ant Tree Clustering}

Social insects display a wide range of collective-level patterns in space and time. Self-assembly behaviors, such as building of structures from a fixed support (a stem or a leaf), have been observed in Linepithema humles Argentina ants and African ants of gender Oecophylla longinoda [5]. Such behavior of biological ants was emulated in [4] to estimate the number of clusters in a given data set. Our work refines the probabilistic behavior of ants towards getting them connected to the tree by introducing a similarity scope for ants and iterative adjustment of the partitions.

Based on the individual interactions and collective self assembling behavior, a number of general rules alongwith a few problem-specific rules can be devised to model artificial ants which build trees to partition the data (see Fig. 1). These are given below:

1. Ants maintain a number of outgoing links towards other ants.

2. Ants maintain an incoming link from other ants.

3. Ants carry feature vectors containing characteristic information about which of the partitions they should belong to.

4. Ants maintain and update threshold values for similarity and dissimilarity.

5. Ants maintain their current position in the tree.

6. Ants may remain disconnected throughout the whole training process.

7. The global scope of similarity for ants should be adjusted iteratively. This global scope remains fixed for all the ants throughout an iteration of data partitioning. A suitable selection of this scope leads to a better partitioning of data.

Artificial ants capable of satisfying these requirements are designed to build ant trees. All immediate children of the tree root node and their descendants should correspond to different classes of textures present in an image.

\subsection{Similarity Estimation}

Similarity estimation is required in order to attach every ant to an appropriate location in the tree. When ant trees are to be used for clustering of high-dimensional data, Euclidean distance can be used to measure similarity between any two ants. The similarity measure $\xi(i, j)$ between ants $i$ and $j$, carrying feature vectors $v^{i}$ and $v^{j}$ respectively, is given by

$$
\xi(i, j)=\rho-\sqrt{\frac{1}{M}\left(\sum_{k=1}^{M}\left(v_{k}^{i}-v_{k}^{j}\right)^{2}\right)},
$$

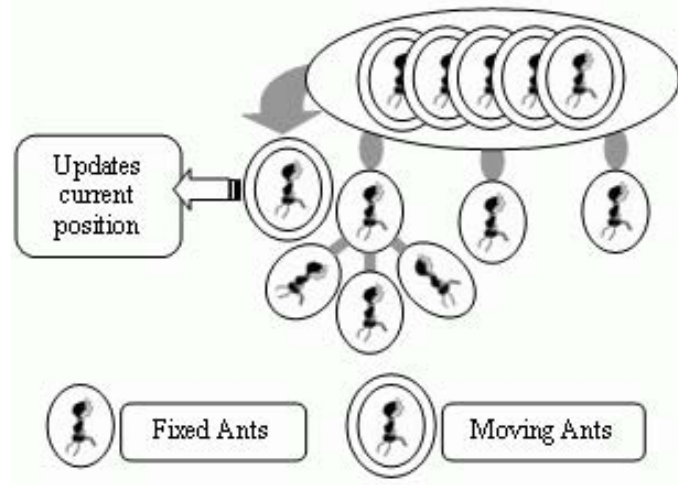

Fig. 1. General principles of tree building

where $M$ is the dimensionality of feature vectors $v$ and $\rho$ is the similarity scope, which initially allows ant $j$ to regard as similar all ants that lie in the range of $\rho / 2$ units.

The key motivation behind the introduction of the similarity scope $\rho$ is the combined effect both $\rho$ and $\beta_{\text {sim }}$, which is a function of $\rho$, can have on partitioning of the feature space. The effect is demonstrated in Fig. 2 for ants carrying 2 -D vectors. Threshold $\beta_{\text {sim }}$ is initialized as $\rho / 2$ for all the ants. Hence the initial least distortion between any two ants directly connected to the root is $\rho$ units which results in a non-overlapping similarity scope of such ants (see Fig. 2). Thus a suitable initial value of $\rho$ is required which may enable the system to completely cover the spread of ants in the vector space. Such a guess may not always be entirely trivial to make. If $\rho$ is very large, the resulting number of partitions may be too small and vice versa. However, a suitable value of $\rho$ can be determined after a number of iterations, as described later in this section.

\subsection{Threshold Update Rules}

Every ant $i$ maintains similarity and dissimilarity threshold values, $\beta_{\text {sim }}(i)$ and $\beta_{\text {dissim }}(i)$ respectively. During the tree construction, some ants may fail to connect to the tree. In that case, the updating of threshold increases their probability of being connected to the tree during the following iteration. Following are the threshold update rules for ant $i$ :

$$
\begin{aligned}
\beta_{\text {sim }}(i) & \leftarrow \beta_{\text {sim }}(i) \times 0.9 \\
\beta_{\text {dissim }}(i) & \leftarrow \beta_{\text {dissim }}(i)+0.01
\end{aligned}
$$

The values for $\beta_{\text {sim }}$ and $\beta_{\text {dissim }}$ are initialized as $\rho / 2$ and 0 respectively, where $\rho$ is the similarity scope.

\subsection{Ant Tree Construction}

The construction of ant tree using artificial ants carrying feature vectors is vital to the clustering of feature data. Ants can 
be arranged in a particular order for addition to the tree. However, we chose not to do so to avoid the extra cost of sorting. Suppose ant $i$ is to be connected to the tree. Starting from the root of the tree, it examines its similarity with the ants that are already connected to the root. If there are no ants already connected to the root, then it connects itself to the root. Otherwise, it finds the most similar ant connected to the root. Following rules are used to define similar and dissimilar ants:

1. If $\xi(i, j)>\beta_{\text {sim }}(i)$, then ant $i$ is similar to ant $j$.

2. If $\xi(i, j)<\beta_{\text {dissim }}(i)$, then ant $i$ is dissimilar to ant $j$.

3. If $\xi(i, j) \geq \beta_{\text {dissim }}(i)$ and $\xi(i, j) \leq \beta_{\text {sim }}(i)$, then ant $i$ is neither similar nor dissimilar to ant $j$ and needs further processing.

If ant $i$ is dissimilar to all the ants currently attached to the root, then it attaches itself to the root. If the root cannot accommodate further ants, then ant $i$ is dropped. If ant $i$ is similar to ant $j$, it moves itself in the direction of ant $j$ and changes it position from root to the ant $j$. During the next iteration, ant $i$ examines its similarity with the children of ant $j$ and repeats the same process till it finds a suitable place. It should be noted that once ant $i$ has moved from the root, it cannot be dropped. If it ends up being dissimilar to all the children ants of its current location, then it moves itself in the direction of the most similar child. In case that ant $i$ is neither similar nor dissimilar to the ant $j$, its similarity and dissimilarity thresholds are updated using (2) and (3) and its processing delayed until next iteration. Threshold update increases the probability of ant $i$ getting connected to ant $j$ in the next iteration if ant $i$ was only marginally short of being similar to ant $j$. On the other hand, threshold update increases the probability of ant $i$ being dissimilar to ant $j$ in the next iteration if it was marginally short of being dissimilar to ant $j$. This is illustrated in Fig. 3 for ants carrying two dimensional feature vectors. The process continues until all the ants are either dropped or have fixed themselves to the tree.

\subsubsection{Iterative Centroid Adjustment}

All immediate children of the tree root are grouped together with their respective descendants to form data partitions. Centroids of all groups (or partitions) are calculated and are used in the following iteration as artificial ants connected to the tree before any other ants. The algorithm stops when the centroids converge. Centroids of the partitions are used for classification purposes in the nearest-centroid sense.

\subsection{Experimental Setup of ATC}

The selection of similarity scope $\rho$ plays an important role for smooth operation of the proposed algorithm. Since a suitable value for $\rho$ cannot always be predicted, two rules are defined to adjust the value of $\rho$. The adjustment rules require a fixed

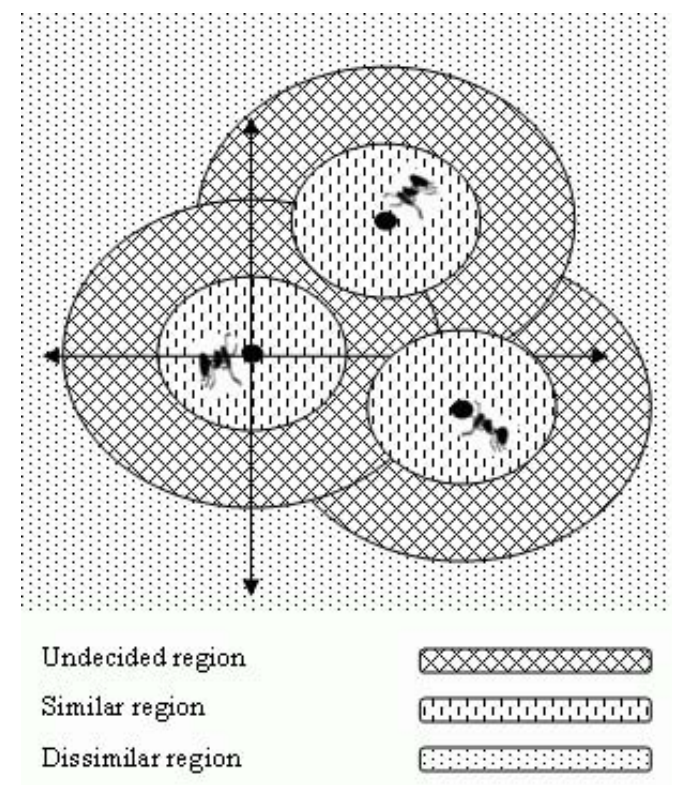

Fig. 2. Partitioning of vector space based on similarity scope $\rho$ and initial similarity threshold value $\rho / 2$ of ants

number of desired partitions $\tau$, which is equal to the number of texture classes present in a given image. If $\rho$ is chosen to be very small, most ants will be dissimilar to ants already attached to the tree and the tree will reach maximum number of children $\tau$ in a fast manner. The value of $\rho$ is updated in the next iteration to allow more ants to be attached to the tree using the following adjustment rule:

$$
\rho=\rho+\frac{\lambda}{\tau} \times \alpha
$$

where $\lambda$ is the number of ants dropped, $\alpha$ is a constant value, and $\tau$ is the maximum number of allowed children of the tree. However, a situation may arise where the number of partitions $n$ is less than $\tau$. To overcome this problem, another rule is defined:

$$
\rho=\rho-\left(\frac{\tau-n}{\tau}\right) \times \alpha
$$

where $n$ is the number of resulting partitions. Equations (4) and (5) together help in iteratively adjusting the value of $\rho$ to a suitable value. The process is repeated until the number of ants being dropped goes below a predefined allowable percentage of dropped ants.

\section{EXPERIMENTAL RESULTS}

We tested the effectiveness of the proposed clustering algorithm using Gabor features on images containing 5 and 16 Brodatz textures [6], some of the de facto standard test images for texture classification [3]. Classification performance was measured by the percentage classification error using the 


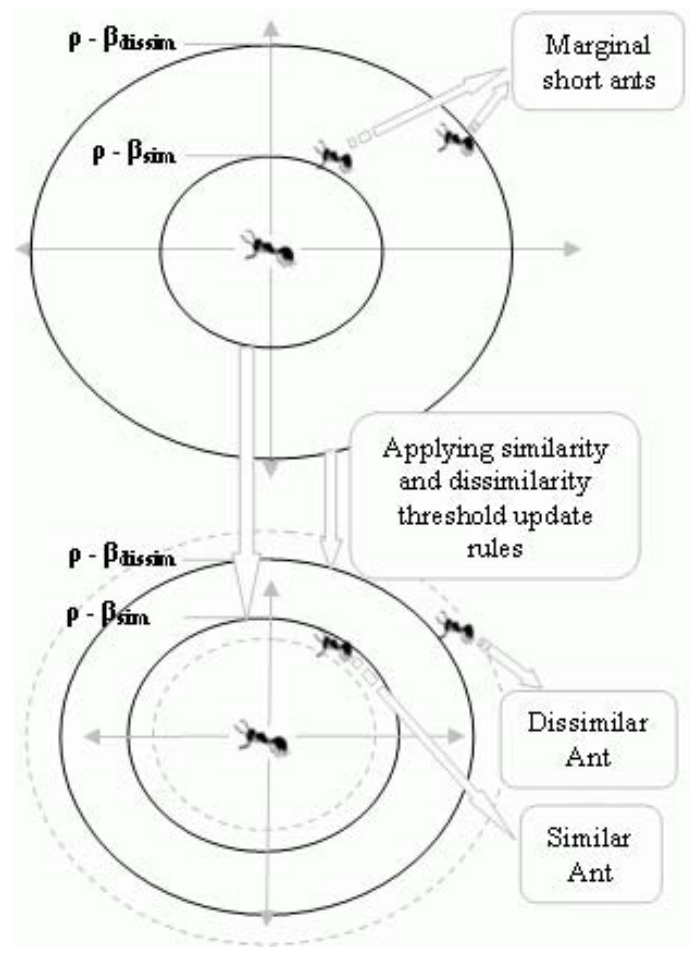

Fig. 3. Effect of applying similarity and dissimilarity threshold for 2-D feature vectors

confusion matrix. The performance of ATC was compared with that of $k$-means clustering algorithm on the same Gabor features, and averages of different methods as reported in [3]. Quantitative results, as given in Table 1, show that the ATC's classification performance compares favorably to that of all the other methods tested. In almost all cases, the classification error using Gabor features is less than the averages of different filtering methods in [3]. ATC also outperforms the $k$-means clustering algorithm in some of the cases, by over $14 \%$ in case of the 16-texture image referred to as Nat-16v by the authors of [3], regarded as one of the most difficult test images. It was observed from the visual results, not included here due to the limited space, that the two clustering algorithms most often have difficulties in similar areas. This might be due to the reason that in such areas, the algorithms do not have sufficient characteristic information from the Gabor features.

\section{CONCLUSIONS}

In this paper, we have presented a novel texture classification algorithm based on ant tree clustering of Gabor features. The clustering process is motivated by the self-assembling behavior of natural ants for forming mechanical structures such as drops, crossing chains, and building chains. Experimental results for classification of multi-texture images reveal the promise carried by the proposed algorithm. While being com-

\begin{tabular}{|l||c|c|c|ccc|}
\hline Image & {$\left[^{\mathbf{3}}\right]^{\dagger}$} & $\boldsymbol{k}$-means & ATC & $\mu_{1}^{*}$ & $\mu_{2}^{*}$ & $\mu_{3}^{*}$ \\
\hline Nat-5c & a & $\mathbf{7 . 9 4}$ & 7.95 & 11.50 & 24.10 & 17.20 \\
\hline Nat-5v & b & $\mathbf{1 4 . 0 7}$ & 14.08 & 27.20 & 44.00 & 33.60 \\
\hline Nat-5v2 & c & 17.00 & $\mathbf{1 6 . 8 8}$ & 25.90 & 38.70 & 33.50 \\
\hline Nat-5v3 & d & 23.77 & $\mathbf{2 3 . 7 1}$ & 31.10 & 41.00 & 33.20 \\
\hline Nat-5m & e & 20.68 & $\mathbf{2 0 . 5 9}$ & 24.70 & 41.10 & 30.30 \\
\hline Nat-16c & f & $\mathbf{3 8 . 0 1}$ & 39.39 & 47.80 & 51.10 & 50.70 \\
\hline Nat-16v & g & 56.78 & $\mathbf{4 4 . 6 0}$ & 54.40 & 62.70 & 58.20 \\
\hline
\end{tabular}

Table 1. Classification error (\%) for some of the test images in [3]

${ }^{*} \mu_{1}, \mu_{2}$, and $\mu_{3}$ denote the average classification error in [3] using the following mixtures of filtering techniques:

$\mu_{1}$ - Heuristically designed texture feature extractors $\mu_{2}$ - Optimized texture feature extractor $\mu_{3}$ - Wavelet, Gabor, and QMF full-rate filter bank $\dagger$ Correspondence to reference images in Fig. 11 in [3]

putationally efficient, the probabilistic nature of the algorithm allows it to avoid local minima which the traditional $k$-means algorithm may get stuck in. Our future work will attempt to improve the clustering performance of the algorithm by employing more robust measures for construction of the ant trees.

\section{REFERENCES}

[1] K. Laws, Textured Image Segmentation, Ph.D. thesis, University of Southern California, USA, 1980.

[2] M. Tuceryan and A. Jain, Handbook of Pattern Recognition and Computer Vision, in Chen, C. and Pau, L. and Wang, P. (Editors), World Scientific, 2nd edition, 1998.

[3] T. Randen and J. Husoy, "Filtering for texture classification: A comparative study," IEEE Transactions on Pattern Analysis and Machine Intelligence, vol. 24, no. 11, pp. 1542-1550, 2002.

[4] H. Azzag, N. Monmarch, M. Slimane, G. Venturini, and C. Guinot, "Anttree: a new model for clustering with artificial ants," in IEEE Congress on Evolutionary Computation, Australia, Dec 2003, vol. 1, pp. 2642-2647.

[5] G. Theraulaz, E. Bonabeau, C. Sauwens, J-L. Deneubourg, A. Lioni, F. Libert, L. Passera, and RV. Sol, "Model of droplet formation and dynamics in the argentine ant (linepithema humile mayr)," Bulletin of Methematical Biology, vol. 63, pp. 1079-1093, 2001.

[6] P. Brodatz, A Photographic Album for Artists and Designers, Dover, New York, 1966. 